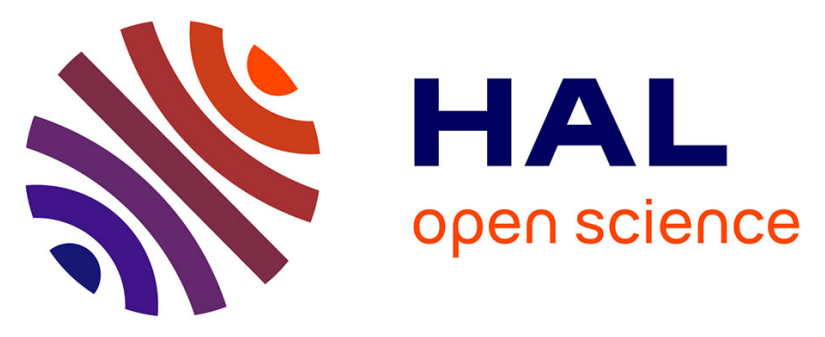

\title{
On Task-Decoupling by Robust Eigenstructure Assignment for Dexterous Manipulation
}

Alex Caldas, Alain Micaelli, Mathieu Grossard, Maria Makarov, Pedro

Rodriguez-Ayerbe, Didier Dumur

\section{- To cite this version:}

Alex Caldas, Alain Micaelli, Mathieu Grossard, Maria Makarov, Pedro Rodriguez-Ayerbe, et al.. On Task-Decoupling by Robust Eigenstructure Assignment for Dexterous Manipulation. IEEE/RSJ International Conference on Intelligent Robots and Systems ( IROS 2015), 2015, HAMBURG, Germany. pp.5654-5661. hal-01259275v2

\section{HAL Id: hal-01259275}

\section{https://hal-centralesupelec.archives-ouvertes.fr/hal-01259275v2}

Submitted on 12 Mar 2020

HAL is a multi-disciplinary open access archive for the deposit and dissemination of scientific research documents, whether they are published or not. The documents may come from teaching and research institutions in France or abroad, or from public or private research centers.
L'archive ouverte pluridisciplinaire HAL, est destinée au dépôt et à la diffusion de documents scientifiques de niveau recherche, publiés ou non, émanant des établissements d'enseignement et de recherche français ou étrangers, des laboratoires publics ou privés. 


\title{
On Task-decoupling by Robust Eigenstructure Assignment for Dexterous Manipulation
}

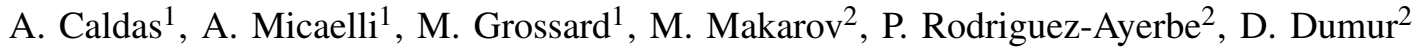

\begin{abstract}
This paper presents a new control scheme for dexterous manipulation of an object by a multifingered hand. The aim of this control is to ensure the tracking by the object of specific trajectories depending on the task. More specifically, we consider a decoupling control according to the motion directions in the task space. The control scheme is based on a new eigenstructure assignment method, where the placement of the closed-loop eigenvalues ensures the stability of the system hand/object, and strict placement of the object-level eigenvectors ensures the system decoupling. A parallel is made between task specifications and synergies. Current synergy controllers are often bio-inspired and based on the analysis of synergies in the human hand. The proposed algorithm allows the specification of precise object level performance according to the task, and to find the related synergies. Moreover, robustness to uncertainties on the contact point is an additional objective of the control law, and the effect of these uncertainties on the decoupling is investigated. Simulation results are presented and illustrate the decoupling effect of the control law on the system hand/object in the 2D-cartesian space.
\end{abstract}

\section{INTRODUCTION}

In the last few decades, robotics has evolved into ever more complex systems working on ever more complex manipulation tasks. From these emerging needs, roboticists have proposed new mechanical designs and multifingered hands have been developed. Various strategies have been proposed to control these mechanical systems and dexterous manipulation is an active field of research [1]-[3].

Among all the proposed approaches, studies on the hand synergies are particularly interesting [4][5]. Synergies reduce the complexity of the control law by correlating the degrees of freedom of the hand, which reduces the dimension of the controller. Most of studies on synergies focus on the problem of grasping an object with a multifingered hand, and just few consider synergistic controllers for dexterous manipulation. In grasping researches, the proposed synergies are bio-inspired and deduced from a human grasp database [6][7]. In dexterous manipulation, an optimal feedback control is applied in [8] and only a subspace of the available control space is utilized according to a given task, i.e. the actuators are synergistically coupled and obeying to the minimal intervention principle [9]. To our knowledge, no

\footnotetext{
${ }^{1}$ A. Caldas and M. Grossard are with the Interactive Robotic Laboratory of CEA-LIST and A. Micaelli is with the Interactive Simulation Laboratory of CEA-LIST 91192 Gif sur Yvette (e-mail: alex.caldas@cea.fr; alain.micaelli@cea.fr; mathieu.grossard@cea.fr).

${ }^{2}$ M. Makarov, P. Rodriguez-Ayerbe and D. Dumur are with Laboratoire des Signaux et Systèmes (UMR 8506) CentraleSupelec - CNRS - Université Paris-Sud, Control Departement, 91192, Gif-sur-Yvette, France (e-mail: maria.makarov@centralesupelec.fr; pedro.rodriguez@centralesupelec.fr; didier.dumur@centralesupelec.fr).
}

other works relate task specifications and synergies with the control law for dexterous manipulation. Moreover, no other works deal with a decoupling objective for the closed-loop hand/object system according to task specifications, as for instance moving the object in the $x$-axis of the 2D cartesian space without affecting the translation along the $y$-axis or the rotation in the $(x, y)$ plan (Fig. 1). In this paper, a control scheme with eigenstructure assignment is proposed, allowing to decouple the system at the object level. The link between task decoupling and synergies is shown.

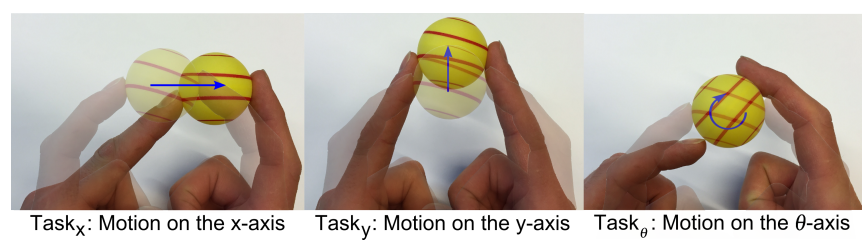

Fig. 1. Example of task specifications.

In control theory, eigenstructure assignment consists in designing a state feedback which places the closed-loop eigenvalues (poles) and eigenvectors (defining the distribution of the modes for each state) of a Multi-Input Multi-Output (MIMO) system at some prescribed locations according to the closed-loop specifications. Most of the eigenstructure assignment methods consider the placement of the closed-loop eigenvectors when the eigenvalues are fixed to predefined values [10]. When only pole placement is needed without any eigenvector assignment, constraints on the system can be defined to place the closed-loop poles in a stable $\mathscr{D}$ region of the complex space [11]. Loose assignment allows to define $\mathscr{D}$-regions to place the poles in the complex space, and cones to define the direction of the eigenvectors [12]. To our knowledge, no algorithm allows to define strict directions for the eigenvectors while placing the eigenvalues in a $\mathscr{D}$-region. In this paper, an optimization problem involving Linear Matrix Inequalities (LMIs) and Bilinear Matrix Inequalities (BMIs) is defined to address this issue and find a statefeedback to control the linearized hand/object MIMO system. This algorithm allows us to ensure the stability of the object motion with the closed-loop pole placement and decouple the system according to task specifications with the eigenvectors assignment.

One difficulty in dexterous manipulation tasks is to deal with uncertainties. To better comply with practical experimental conditions, the proposed control law for dexterous manipulation can be improved by including considerations about geometric uncertainties on the contact point based on 
[13].

To sum up, the present paper proposes:

- A control law which ensures the stability of the object motion and decoupling the system according to task specifications.

- A new eigenstructure assignment algorithm allowing to place the eigenvectors without a strict assignment of the eigenvalues.

- Robustness to uncertainties on the contact point which can be specified in the algorithm.

The paper is organized as follows: in section II, a statespace representation of the system is presented. Section III describes the proposed control law. Numerical examples and simulations of the controller are presented in section IV. Section $\mathrm{V}$ gives the conclusions and perspectives.

\section{MULTIFINGERED HAND REPRESENTATION}

This part presents the multifingered hand model, based on the screw theory [14], and introduces useful notations.

\section{A. State-Space Formulation}

The multifingered hand model is obtained by combining the dynamics of the fingers and the object:

$\left\{\begin{array}{l}M_{h}(q) \ddot{q}+C_{h}(q, \dot{q}) \dot{q}+N_{h}(q, \dot{q})-J_{h}\left(q, x_{o}\right)^{T} f_{c}=\tau \\ M_{o}\left(x_{o}\right) \ddot{x}_{o}+C_{o}\left(x_{o}, \dot{x}_{o}\right) \dot{x}_{o}+N_{o}\left(x_{o}, \dot{x}_{o}\right)+G f_{c}=0\end{array}\right.$

- For the first equation, $q=\left[\begin{array}{llll}q_{f_{1}} & q_{f_{2}} & \ldots & q_{f_{k}}\end{array}\right]^{T} \in \mathbb{R}^{n_{q}}$ is the actuated joint position vector for the $k$ fingers of the hand, $q_{f_{i}} \in \mathbb{R}^{n_{f_{i}}}$ being the actuated joint positions of the finger $i, \tau \in \mathbb{R}^{n_{q}}$ is the corresponding torque vector, and $M_{h}, C_{h}$ and $N_{h}$ are respectively the stacked inertia matrix, Coriolis, centrifugal and viscous friction matrix and gravity vector of the $k$ fingers of the hand, $J_{h} \in$ $\mathbb{R}^{n_{c} \times n_{q}}$ the hand Jacobian (with $n_{c}$ the dimension of the contact frame), and $f_{c} \in \mathbb{R}^{n_{c}}$ the contact forces applied to the object.

- For the second equation, $x_{o} \in \mathbb{R}^{n_{o}}$ a local coordinate of the object, with $n_{o}=6$ in $3 \mathrm{D}\left(n_{o}=3\right.$ in $\left.2 \mathrm{D}\right)$ the dimension of the object coordinate, $M_{o} \in \mathbb{R}^{n_{o} \times n_{o}}$ the inertia matrix, $C_{o} \in \mathbb{R}^{n_{o} \times n_{o}}$ the Coriolis and centrifugal matrix, $N_{o} \in \mathbb{R}^{n_{o}}$ the gravity vector, $G \in \mathbb{R}^{n_{o} \times n_{c}}$ the grasp map relating the contact frames to the object frame.

The grasping of the object by the fingers is defined by the following constraint:

$$
J_{h}\left(q, x_{o}\right) \dot{q}=G^{T} \dot{x}_{o}
$$

According to (1)-(2), the system motion can be formulated at the object level as follows (see [14] for more details):

$$
M\left(q, x_{o}\right) \ddot{x}_{o}+C\left(q, x_{o}, \dot{q}, \dot{x}_{o}\right) \dot{x}_{o}+N\left(q, x_{o}, \dot{q}, \dot{x}_{o}\right)=G J_{h}^{-T} \tau
$$

with:

$$
\begin{aligned}
M & =M_{o}+G J_{h}^{-T} M_{h} J_{h}^{-1} G^{T} \\
C & =C_{o}+G J_{h}^{-T} C_{h} J_{h}^{-1} G^{T}+G J_{h}^{-T} M_{h} \frac{d}{d t}\left(J_{h}^{-1} G^{T}\right) \\
N & =N_{o}+G J_{h}^{-T} N_{h}
\end{aligned}
$$

In the following, the dependency on $q, \dot{q}, x_{o}, \dot{x}_{o}$ is dropped in the notations for ease of reading. Equations (2)-(6) are valid under the following assumptions:

A1 The system is not redundant, i.e. there is no internal movement of the fingers for a fixed position of the object. In this case, the hand Jacobian $J_{h}$ is square and invertible. This assumption could be relaxed with a model taking into account the joint redundancy [14].

A2 The contact points are fixed, which implies that $G$ is constant. This assumption is naturally made with (2), and will be relaxed later in the paper by taking into consideration the uncertainties on the contact point location.

A3 The contact forces remain in the friction cone. This requirement must be satisfied by the control law.

A4 The grasp is manipulable, i.e. the desired motion can be generated by the fingers. In this case, the hand Jacobian is full row rank [14].

A5 The influence of the gravity term $N$ is negligible or compensated by the control law.

The linearization of (3) around an equilibrium point $\left[\begin{array}{ll}x_{o_{e q}} & \dot{x}_{o_{e q}}\end{array}\right]^{T}$ leads to an LTI state-space representation [14]:

$$
\frac{d}{d t}\left[\begin{array}{c}
\tilde{x}_{o} \\
\dot{\tilde{x}}_{o}
\end{array}\right]=\left[\begin{array}{cc}
\mathbb{O}_{n_{o} \times n_{o}} & \mathbb{I}_{n_{o} \times n_{o}} \\
\mathbb{O}_{n_{o} \times n_{o}} & -M_{e q}^{-1} C_{e q}
\end{array}\right]\left[\begin{array}{c}
\tilde{x}_{o} \\
\dot{\tilde{x}}_{o}
\end{array}\right]+\left[\begin{array}{c}
\mathbb{O}_{n_{o} \times n_{q}} \\
M_{e q}^{-1} G J_{h_{e q}}^{-T}
\end{array}\right] \tau
$$

where constant matrices $M_{e q}, C_{e q}$ and $J_{h_{e q}}$ define respectively the inertia matrix, Coriolis and centrifugal matrix, and the hand Jacobian calculated at the equilibrium point. $\tilde{x}_{o}$ represents variation of $x_{o}$ around the equilibrium point. Equations (7) and (2) are valid at the equilibrium point $x_{o_{e q}}$ and $q_{e q}$.

The control inputs of the proposed state-space representation (7) are the joint torques $\tau$. These signals can be expressed at the object level, allowing a direct connection between the object position error and the joint torques expressed at the object level. The transform between the object and the joint spaces depends on the hand Jacobian $J_{h}$ and the grasp map $G$, and can be expressed by:

$$
\tau=\underbrace{\hat{J}_{h_{e q}}^{T} G^{+} u}_{\tau_{\text {motion }}}+\underbrace{\hat{J}_{h_{e q}}^{T} N_{G} \lambda}_{\tau_{\text {internal }}}
$$

where $\hat{J}_{h_{e q}}$ is the estimate of the hand Jacobian at the equilibrium point, and $u$ the control input at the object level, $(.)^{+}$defines the pseudo-inverse, which is not unique for the grasp map $G$. The term $N_{G} \lambda \in \operatorname{Ker}(G)$, with $N_{G}$ a base of the null space of $G$, is introduced, expressing the non-uniqueness of the pseudo-inverse, and introducing a degree of freedom $\lambda$, that can be used to fulfill the constraints A3.

The transform $J_{h_{e q}}$ links the joint level to the contact level and the transform $G$ links the contact level to the object level. The first contribution $\tau_{\text {motion }}$ generates the object motion, expressed at the object level, and the second contribution $\tau_{\text {internal }}$ generates internal forces, which do not generate movement in the nominal case, but introduce an additional degree of freedom to fulfill the constraints. This 
decomposition of the torque vector is well known and was proposed since the 1980s [15].

Injecting (8) in (7) leads to:

$$
\begin{aligned}
\frac{d}{d t}\left[\begin{array}{c}
\tilde{x}_{o} \\
\dot{\tilde{x}}_{o}
\end{array}\right]= & {\left[\begin{array}{cc}
\mathbb{O}_{n_{o} \times n_{o}} & \mathbb{I}_{n_{o} \times n_{o}} \\
\mathbb{O}_{n_{o} \times n_{o}} & -M_{e q}^{-1} C_{e q}
\end{array}\right]\left[\begin{array}{c}
\tilde{x}_{o} \\
\dot{\tilde{x}}_{o}
\end{array}\right] } \\
& +\left[\begin{array}{c}
\mathbb{O}_{n_{o} \times n_{q}} \\
M_{e q}^{-1} G J_{h_{e q}}^{-T}
\end{array}\right] \hat{J}_{h_{e q}}^{T} G^{+} u+\left[\begin{array}{c}
\mathbb{O}_{n_{o} \times n_{q}} \\
M_{e q}^{-1} G J_{h_{e q}}^{-T}
\end{array}\right] \hat{J}_{h_{e q}}^{T} N_{G} \lambda
\end{aligned}
$$

If the estimation of the hand Jacobian is perfect, i.e. $\hat{J}_{h_{e q}}=$ $J_{h_{e q}}$, the model becomes:

$$
\frac{d}{d t}\left[\begin{array}{c}
\tilde{x}_{o} \\
\dot{\tilde{x}}_{o}
\end{array}\right]=\underbrace{\left[\begin{array}{cc}
\mathbb{O}_{n_{o} \times n_{o}} & \mathbb{I}_{n_{o} \times n_{o}} \\
\mathbb{O}_{n_{o} \times n_{o}} & -M_{e q}^{-1} C_{e q}
\end{array}\right]}_{A}\left[\begin{array}{c}
\tilde{x}_{o} \\
\dot{\tilde{x}}_{o}
\end{array}\right]+\underbrace{\left[\begin{array}{c}
\mathbb{O}_{n_{o} \times n_{o}} \\
M_{e q}^{-1}
\end{array}\right]}_{B} u
$$

In this specific case, the object motion is only affected by the control input $u$. Equation (10) will be used for the design of the control law.

\section{B. Model of the Contact Uncertainties}

Uncertainties on the contact point are due to modeling errors and can be of different types [13], which affects the grasp map $G$ as follows:

$$
G=G(\delta)
$$

with $\delta \in P_{\delta}$ the vector of uncertainties. $\delta$ can be structured as a translation and/or a rotation error, linear or nonlinear, with $P_{\delta}$ being the set of all the uncertainties considered in the system (linear dependence of $G$ on $\delta$ is investigated in [16]).

\section{CONTROL SCHEME}

Before presenting the control law, the task specifications are formulated using invariant subspaces and related to synergies. The objectives of the control law and the eigenstructure assignment are then presented.

A. Task Specifications, Invariant Subspaces and Synergies

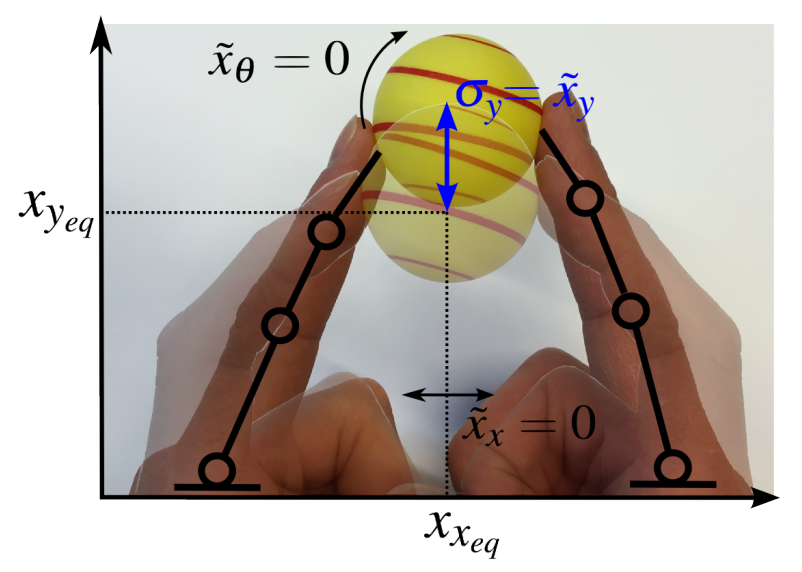

Fig. 2. Task specification: motion only on $y$-axis.

A task specification can be described as a motion of the object, that can be mathematically defined as a subspace of the object space. To clarify, we take an example in the $2 \mathrm{D}$ cartesian space, In this case, the object-level positions are:

$$
\tilde{x}_{o}=\left[\begin{array}{lll}
\tilde{x}_{x} & \tilde{x}_{y} & \tilde{x}_{\theta}
\end{array}\right]^{T}
$$

with $\tilde{x}_{x}, \tilde{x}_{y}$ and $\tilde{x}_{\text {theta }}$ the difference between the equilibrium point and the actual position in respectively the $x$-axis, the $y$-axis and the orientation $\theta$ of the object.

Let us consider a case where the task consists in moving the object through the $y$-axis without displacement through the $x$-axis and without rotating (Fig. 2). Only $\tilde{x}_{y}$ should be affected by this motion but not the other positions. This decoupling objective can be defined as a subspace of the object space:

$$
\left[\begin{array}{c}
\tilde{x}_{x} \\
\tilde{x}_{y} \\
\tilde{x}_{\theta}
\end{array}\right] \in \operatorname{Task}_{y} \Leftrightarrow\left[\begin{array}{c}
\tilde{x}_{x} \\
\tilde{x}_{y} \\
\tilde{x}_{\theta}
\end{array}\right]=\underbrace{\left[\begin{array}{l}
0 \\
1 \\
0
\end{array}\right]}_{S b_{y}} \sigma_{y}
$$

with $\sigma_{y}$ the magnitude of the movement, $S b_{y}$ the subspace of the object space selecting the $\tilde{x}_{y}$ state.

Thus, a task can be described as a subspace of the object space. Specifications on the velocities can be added by considering $\dot{\sigma}$ (as in section IV). To ensure a perfect trajectory tracking of the task, its corresponding subspace should be orthogonal to any other movement subspaces with respect to the closed-loop hand/object system. This specification can be defined as follows:

$$
\overline{S b}^{T} A_{C L} S b=0
$$

with $\overline{S b}$ the complement set of $S b$ in the object space, and $A_{C L}$ the state space matrix of the closed-loop system hand/object.

Let us note that a parallel can also be made with synergies. The joint displacement vector $q$ can be represented as a function of fewer elements, collected in a synergy intensity vector $\sigma$, which effectively constrain hand configuration in a $n_{s}$-dimensional manifold [4]:

$$
q=S \sigma
$$

with $S \in \mathbb{R}^{n_{q} \times n_{s}}$ the matrix of synergies, with $n_{s}$ the number of synergies.

To clarify the concept of the synergies, we consider the example in Fig. 2. The joint angles of the hand can be linked to apply the movement of the object along the $y$-axis. This motion is a subspace of the object space and in the linear case, the transform $J_{h_{e q}}^{-1} G^{T}$ links the joint level to the object level, so this motion is a subspace of the joint space, and deducing from (13):

$$
q \in \operatorname{Task}_{y} \Leftrightarrow q=\underbrace{\left(J_{h_{e q}}^{-1} G^{T}\right)^{+} S b_{y}}_{S_{y}} \sigma_{y}
$$

Thus, $S_{y}$ can be one line of the matrix $S$ and $\sigma_{y}$ can be one component of the synergy intensity vector $\sigma$, and the synergy space can be specified from task objectives. 


\section{B. Objectives of the Control Law}

With respect to the proposed control input (8), the control objectives are:

- Stability of the object. This objective consists in placing the eigenvalues of the closed-loop system in a stable region of the complex space.

- Trajectory tracking of the object. As seen in III-A, this objective can be achieved by decoupling the system according to the task specifications, by including the constraints (14) in the specification of the controller. The constraints can be fulfilled by placing the closedloop eigenvectors.

- Satisfying the system constraints, e.g. ensuring that the contact forces remain in the friction cone. This objective is achieved with the control input $\tau_{\text {internal }}$ that generates internal forces. In this paper, this part of the control law is not considered (see [13] for more details).

- The previous objectives must be performed in presence of contact uncertainties. The proposed control design can be applied for a set of systems, which is equivalent to achieve the previous objectives for the system affected by a set of uncertainties.

In the view of the previous objectives, we choose a control law by static feedback gain which will be designed by eigenstructure assignment (Fig. 3).

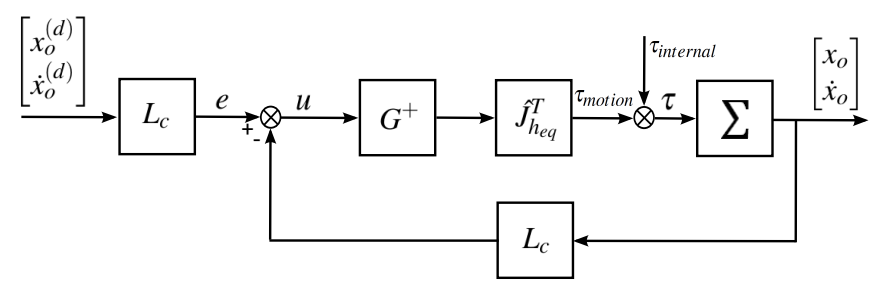

Fig. 3. General control scheme.

\section{Eigenvalue Placement}

As seen before, the motion control is ensured by the signal $u$. The proposed state feedback is:

$$
u=e-L_{c} x
$$

with $e$ the input of the closed-loop system and $L_{c}$ the state feedback matrix.

The closed-loop state-space equation becomes:

$$
\dot{x}=\left(A-B L_{c}\right) x+B e
$$

with $A$ and $B$ from (10).

The object motion dynamics is specified by pole placement, i.e. by choosing the eigenvalues of the matrix $A-B L_{c}$. This can be achieved by defining a stable $\mathscr{D}$-region in the complex space. Classically, three different forms can be used to specify the stable $\mathscr{D}$-region [11]: the left half-plane, the cone and the disc (Fig. 4).

The $\mathscr{D}$-region can be described by LMI constraints depending on $P=P^{T} \succ 0$ a square positive-definite matrix (" $\succ$ " stands for positive definite), which defines the Lyapunov stability, $A$ the state matrix to stabilize, $Y=L_{c} P$ defining the state feedback, and the parameters $\alpha, r$ and $\Phi$ defining respectively the left half-plane, the disc and the cone [11]. The LMI problem consists in finding $P$ and $Y$ under the following constraints:

- The left half-plane defined by $\alpha$ :

$$
(A P-B Y)+\left(P A^{T}-Y^{T} B^{T}\right)+2 \alpha P \prec 0
$$

The choice of $\alpha>0$ avoids instability and sets minimal dynamics convergence.

- The disc centered in 0 with a radius $r$ :

$$
\left[\begin{array}{cc}
-r P & A P-B Y \\
P A^{T}-Y^{T} B^{T} & -r P
\end{array}\right] \prec 0
$$

The disc radius is chosen according to the maximal dynamics of the system, e.g. due to the hardware sampling period constraint or minimal noise influence.

- The cone centered in 0 with an angle $\Phi$ (with $X^{\prime}=$ $A P-B Y)$ :

$$
\left[\begin{array}{cc}
\sin (\Phi)\left(X^{\prime}+X^{\prime T}\right) & \cos (\Phi)\left(X^{\prime}-X^{\prime T}\right) \\
\cos (\Phi)\left(X^{\prime T}-X^{\prime}\right) & \sin (\Phi)\left(X^{\prime}+X^{\prime T}\right)
\end{array}\right] \prec 0
$$

This sector allows to constrain the damping ratio.

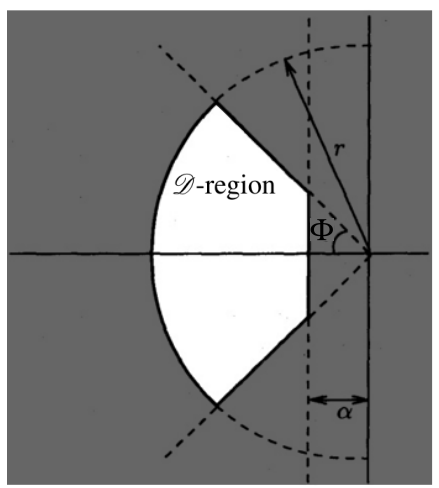

Fig. 4. LMI Regions [11].

The pole placement can be achieved by solving the feasibility problem: finding $P$ and $Y$ such that (19) to (21) are true. Then, the state-feedback matrix is deduced:

$$
L_{c}=Y P^{-1}
$$

\section{Eigenstructure Assignment}

The eigenstructure assignment aims to place the eigenvalues and the eigenvectors of the closed-loop system. As seen in the previous section, the placement of the eigenvalues ensure the stability of the system, and the eigenvectors, the last degree of freedom in the feedback synthesis, is used to fulfill the second objective: decoupling the system according to task specifications. This can be done by defining invariant subspaces $S b_{i}$ related to the task specifications and the problem can be expressed as follows:

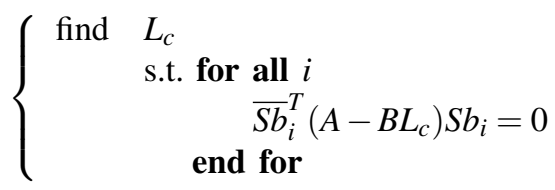


(23) means the subspaces $S b_{i}$ are invariant through $A-B L_{c}$, but does not ensure a stable feedback control.

To solve the stability issue, the pole placement with (19)(21) and the decoupling (23) should be satisfied under the constraint $Y=L_{c} P$. Thus, this problem can be solved by:

$$
\left\{\begin{array}{rlr}
\min _{Y, P, L_{c}} \varepsilon & \\
& \text { s.t. }> & (19)-(21) \text { are satisfied } \\
& & \text { for all } i \\
& & \overline{S b}_{i}^{T}\left(A-B L_{c}\right) S b_{i}=0 \\
& & \text { end for } \\
& > & -\varepsilon \leq Y-L_{c} P \leq \varepsilon
\end{array}\right.
$$

The first constraint finds a controller $Y P^{-1}$ that ensures the stability of the closed-loop system, the for-loop finds a controller $L_{c}$ that ensures the decoupling of the subspaces, and the last constraint ensures the convergence between $L_{c}$ and $Y P^{-1}$. This problem is nonlinear and the algorithm contains LMIs and BMIs.

\section{E. Algorithm Coping with Uncertainties}

If uncertainties affect the system linearly, the classical method could be to write it in a Linear Parametric Varying (LPV) form. Here, the uncertainties on the contact points affect the grasp map, and the system representation (10) is not linear according to the matrix $G$, thus it is not straightforward to formulate the model as a LPV system. Instead, the following approach is used.

A set $P_{\Sigma}$ of all possible state-space representations $\Sigma=$ $(A, B, C, D)$ depending on the uncertainties is defined:

$$
\Sigma \in P_{\Sigma} \Leftrightarrow\left\{\forall \delta \in P_{\delta}, \Sigma:=\{\dot{x}=A(\delta) x+B(\delta) u\}\right\}
$$

Knowing that the uncertainties do not affect linearly this set, no assumption can be made about the convexity of $P_{\Sigma}$. The uncertainties can be taken into account by discretizing the set $P_{\delta}$, implying a discretization of $P_{\Sigma}$ and defining set of systems that is called thereafter discretized- $P_{\Sigma}$ systems.

As the state matrix $A(\delta)$ varies according to the uncertainties, it is impossible to find decoupled subspaces for all the discretized- $P_{\Sigma}$ systems. Therefore, the algorithm proposed here considers invariant subspaces $S b_{i}$ only with the system unaffected by uncertainties, but stability is ensured for all the discretized-P $P_{\Sigma}$ systems. The optimization problem can be formulated as follows:

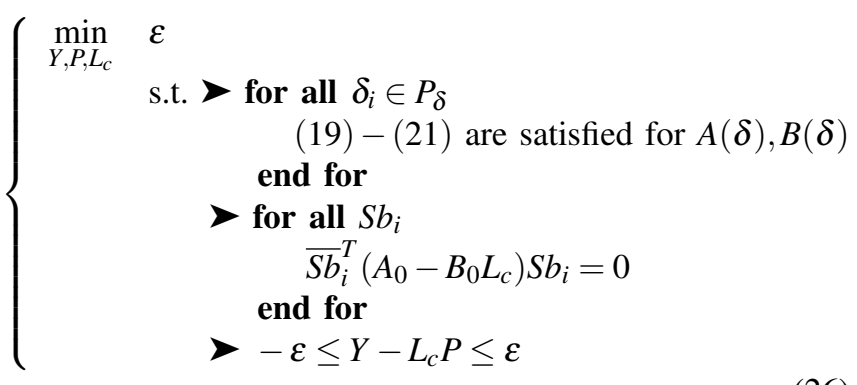

The first for-loop finds a controller $Y P^{-1}$ that ensures the stability of all the discretized- $P_{\Sigma}$ systems, the second forloop finds a controller $L_{c}$ that ensures the decoupling of the subspaces for the nominal system $\left(A_{0}, B_{0}\right)$, and the last constraint ensures the convergence between $L_{c}$ and $Y P^{-1}$.

\section{NUMERICAL EXAMPLES AND SIMULATIONS}

This section presents the simulation results of the proposed control scheme for different examples. Simulations are done

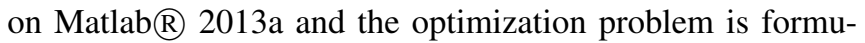
lated with the YALMIP toolbox using the SeDuMi 1.3 and PENBMI 2.1 solvers [17].

\section{A. 2D uncertain manipulation system}

The following 2D example relates to a grasp with two contact points. The contact forces $f_{c}=\left[f_{c_{1 x}} f_{c_{1 y}} f_{c_{2 x}} f_{c_{2 y}}\right]^{T} \in$ $\mathbb{R}^{4}$ have to satisfy the following Coulomb friction constraints (Fig. 5):

$$
\left(\begin{array}{cccc}
-1 & -\mu & 0 & 0 \\
1 & -\mu & 0 & 0 \\
0 & 0 & -1 & -\mu \\
0 & 0 & 1 & -\mu
\end{array}\right)\left(\begin{array}{l}
f_{c_{1_{x}}} \\
f_{c_{1_{y}}} \\
f_{c_{2_{x}}} \\
f_{c_{2_{y}}}
\end{array}\right)<\left(\begin{array}{l}
0 \\
0 \\
0 \\
0
\end{array}\right)
$$

with the friction coefficient $\mu=1$. The grasp map is:

$$
G_{0}=\left(\begin{array}{cccc}
0 & -1 & 0 & 1 \\
1 & 0 & -1 & 0 \\
r_{o} & 0 & r_{o} & 0
\end{array}\right)
$$

with the length of the rectangular object fixed to $r_{o}=20 \mathrm{~mm}$. Two types of uncertainties are considered:

E1 Translation error (Fig. 5a). The direction of the contact force is considered as known, but the location of the contact point is uncertain. The grasp map becomes:

$$
G_{r}=\left(\begin{array}{cccc}
0 & -1 & 0 & 1 \\
1 & 0 & -1 & 0 \\
r_{o} & 0 & r_{o} & \delta
\end{array}\right)
$$

where $\delta= \pm 5 \mathrm{~mm}$ represents the translation uncertainty of the second contact point.

E2 Orientation error (Fig. 5b). The location of the contact point is considered as known but the orientation of the contact force is uncertain. The grasp map becomes:

$$
G_{r}=\left(\begin{array}{cccc}
0 & -1 & \sin \left(\theta_{\delta}\right) & \cos \left(\theta_{\delta}\right) \\
1 & 0 & \cos \left(\theta_{\delta}\right) & \sin \left(\theta_{\delta}\right) \\
r_{o} & 0 & r_{o} \cos \left(\theta_{\delta}\right) & -r_{o} \sin \left(\theta_{\delta}\right)
\end{array}\right)
$$

with $\theta_{\delta}= \pm 10^{\circ}$ being the orientation error of the second contact point.

\section{B. Control Design}

The motion control was designed with the following $\mathscr{D}$ region parameters (first objective):

- $\alpha=0.4$, which ensures $\operatorname{Re}($ pole $)<-0.4$, providing a stability constraint and minimal dynamics with maximum overshoot at 7.5 seconds for the slowest modes.

- $\Phi=0.52 \mathrm{rad}$, which guarantees a minimum damping ratio of $\xi \simeq 0.86$.

- $r=7$, which sets the maximal dynamics with maximum overshoot at 0.43 seconds for the fastest modes. 
a) Translation error

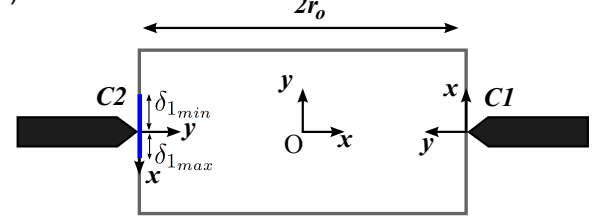

b) Rotation error

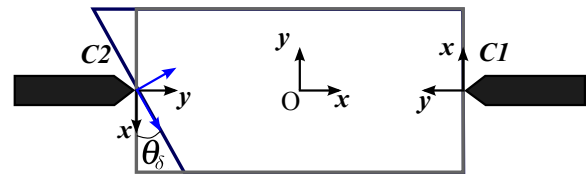

Fig. 5. Proposed example: a) translation error and b) rotation error

The second proposed objective consists in decoupling the movement in the cartesian space. Researches in neurosciences have proved that human reaching movements tend to follow roughly straight lines in the cartesian space [18], so decoupling the movement in this space seems to be a natural way to manipulate dexterously. Moreover, sensitivity to uncertainties is minimized when state decoupling is searched in linear systems [10]. The corresponding subspaces are:

$$
S b_{x}=\left[\begin{array}{ll}
1 & 0 \\
0 & 0 \\
0 & 0 \\
0 & 1 \\
0 & 0 \\
0 & 0
\end{array}\right] ; S b_{y}=\left[\begin{array}{cc}
0 & 0 \\
1 & 0 \\
0 & 0 \\
0 & 0 \\
0 & 1 \\
0 & 0
\end{array}\right] ; S b_{\theta}=\left[\begin{array}{ll}
0 & 0 \\
0 & 0 \\
1 & 0 \\
0 & 0 \\
0 & 0 \\
0 & 1
\end{array}\right]
$$

In each subspace $S b_{i}$, the position is related to its corresponding velocity and decorrelated from the other axis positions and velocities.

\section{Results}

The algorithm (26) is tested for E1 and E2. A solution can be found in 3 to 45 seconds (the algorithm is applied offline), and multistart evaluation should be done to find the best decoupling system. In $\mathrm{E} 1$ and $\mathrm{E} 2, \varepsilon$ is minimized to $10^{-13}$.

First, the controller obtained with the algorithm (26) is tested on the nominal system (linearized system without uncertainties) (Fig. 7). Different movements are applied to the system: a combined movement (d), a movement on the $x$-axis (a), on the $y$-axis (b), and a last one with just a rotation of the object (c). This simulation shows that the system is stable and that the motions are perfectly decoupled in the cartesian space.

Secondly, the control law is tested on the nonlinear system where only a motion on the $x$-axis is specified (Fig. 8 and 6). The first simulation (blue curve in Fig.8 and 6.a) is achieved without uncertainties on the contact point. Both show the effect of the linearization on the decoupled system. When the system moves away from the equilibrium point, the decoupling is less efficient. In Fig. 6.a, we see clearly that near the equilibrium point, the motion is performed along the $x$-axis only, but when the system approaches its final desired
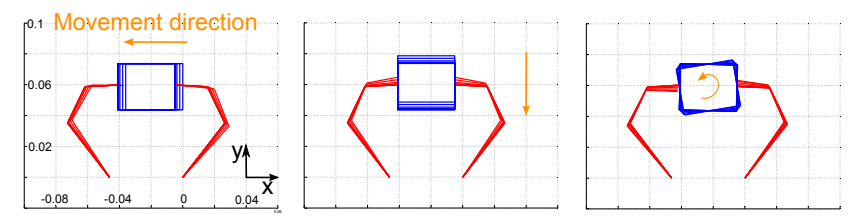

a) Movement on the $x$-axis b) Movement on the $y$-axis c) Movement on the $\theta$-axis Subspace $\mathrm{Sb}_{\mathrm{x}}$
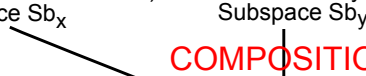

Subspace $\mathrm{Sb}_{\theta}$

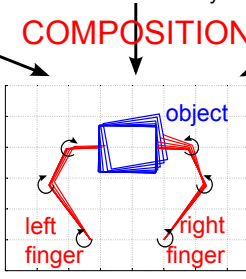

d) Combined movement

Fig. 7. Evaluation of the decoupling on the linearized system without uncertainties

position, it is affected by a re-orientation and a motion on the $y$-axis.

The second and third simulations (dashed red and dotted green lines in Fig.8 and 6.b-c) are done in presence of uncertainties on the contact points (E1 and E2). In Fig.6.b, the contact surface is affected by the uncertainties and in Fig.6.c, the contact point is uncertain. The two examples affect the system decoupling. Fig. 8 shows that orientation and motion on the $y$-axis diverge from zero sooner than when the system in not affected by uncertainties. Thus, the decoupling becomes less efficient sooner in presence of uncertainties. However, the error along the $y$-axis is smaller than $4 \mathrm{~mm}$ for a translation of $30 \mathrm{~mm}$ in the $x$-axis, and the error in rotation is less than $0.2 \mathrm{rad}$. This result could be expected since the algorithm considers only the decoupling of the nominal system.

However, even in presence of uncertainties, the object motion is always stable, as specified in the design of the controller.
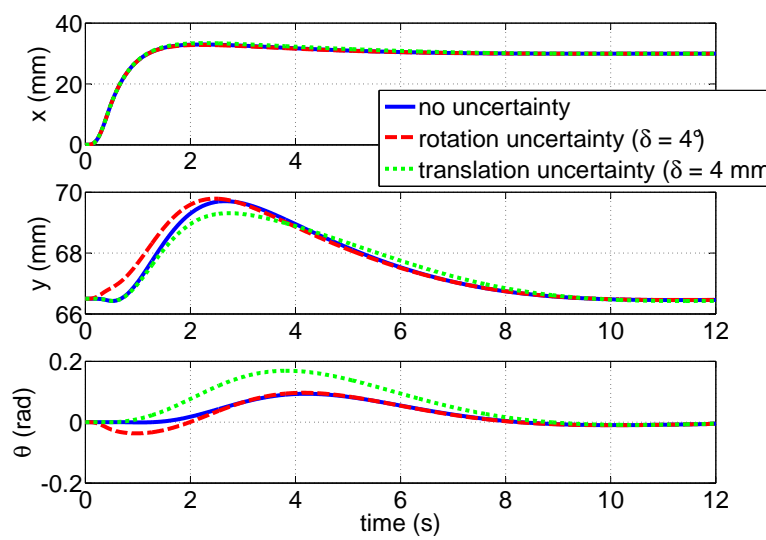

Fig. 8. Time-domain evaluation of the control law on the nonlinear system for a movement on the $x$-axis (see Fig. 6) 


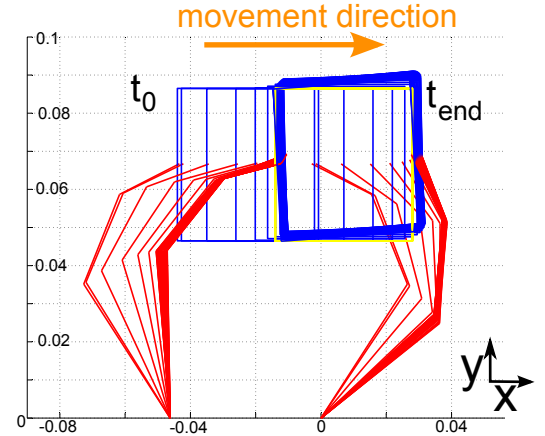

a) No uncertainties

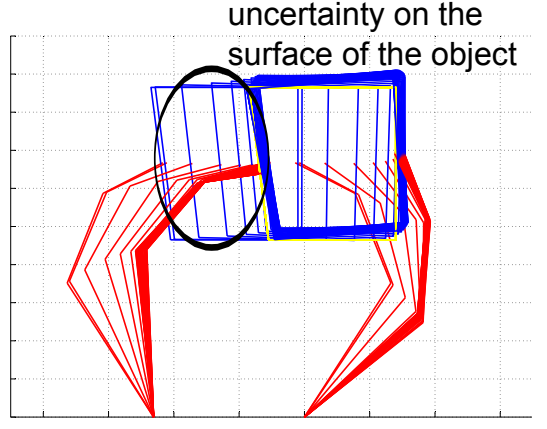

b) Rotation Uncertainty

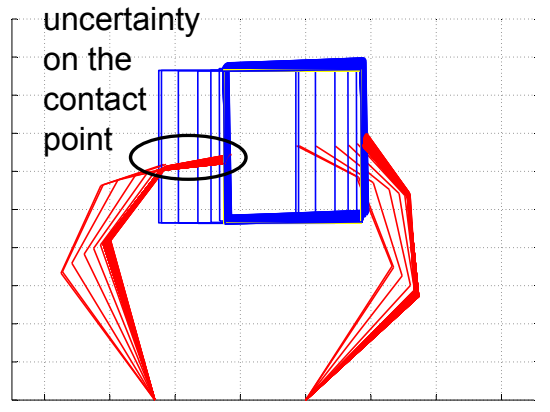

c) Translation Uncertainty

Fig. 6. Influence of uncertainties on the trajectory tracking.

\section{CONCLUSIONS}

The paper presents a new control law that enables trajectory tracking according to task specifications. The objectives at the object level are related to invariant subspaces through the closed-loop system and the link with the synergies is shown. From these relations, the design of a controller is proposed based on a new eigenstructure assignment, where the pole placement allows to stabilize the system hand/object with given closed-loop dynamics and robustness properties, and the eigenvectors placement allows to decouple the system.

Results show a perfect decoupling for the linearized system. When evaluating on the complete nonlinear system, the effect of the linearization becomes visible and the decoupling becomes less efficient when the system moves away from the equilibrium point or when it is affected by uncertainties. Next work should include nonlinearities in the formulation of the decoupling problem or consider different linearized points. However, stability is always ensured, even in presence of uncertainties.

Next step will be in the implementation and evaluation of the proposed controller in a practical case.

\section{REFERENCES}

[1] J. Martin and M. Grossard, "Design of a fully modular and backdrivable dexterous hand," The International Journal of Robotics Research (IJRR) Special Issue on 'Mechanics and Design of Robotic Hands', vol. 33, no. 5, pp. 783-798, 2014.

[2] A. Bicchi, "Hands for dexterous manipulation and robust grasping: A difficult road toward simplicity," IEEE Transactions on Robotics and Automation, vol. 16, no. 6, pp. 652-662, 2000.

[3] A. M. Okamura, N. Smaby, and M. R. Cutkosky, "An overview of dexterous manipulation," in IEEE International Conference on Robotics and Automation, 2000, vol. 1. IEEE, 2000, pp. 255-262.

[4] M. Gabiccini, A. Bicchi, D. Prattichizzo, and M. Malvezzi, "On the role of hand synergies in the optimal choice of grasping forces," Autonomous Robots, vol. 31, no. 2-3, pp. 235-252, 2011.

[5] M. Gabiccini, E. Farnioli, and A. Bicchi, "Grasp analysis tools for synergistic underactuated robotic hands," The International Journal of Robotics Research, 2013.

[6] M. Santello, M. Flanders, and J. F. Soechting, "Postural hand synergies for tool use," The Journal of Neuroscience, vol. 18, no. 23, pp. 10105 $10115,1998$.

[7] T. Wimbock, B. Jahn, and G. Hirzinger, "Synergy level impedance control for multifingered hands," in International Conference on Intelligent Robots and Systems (IROS). IEEE, 2011, pp. 973-979.
[8] E. Todorov and M. I. Jordan, "Optimal feedback control as a theory of motor coordination," Nature neuroscience, vol. 5, no. 11, pp. 1226$1235,2002$.

[9] E. Todorov and Z. Ghahramani, "Analysis of the synergies underlying complex hand manipulation," in Annual International Conference of the Engineering in Medicine and Biology Society, vol. 2. IEEE, 2004, pp. 4637-4640.

[10] J. Kautsky, N. K. Nichols, and P. Van Dooren, "Robust pole assignment in linear state feedback," International Journal of Control, vol. 41, no. 5, pp. 1129-1155, 1985.

[11] M. Chilali and P. Gahinet, "Hinf design with pole placement constraints: an LMI approach," IEEE Transactions on Automatic Control, vol. 41, no. 3, pp. 358-367, 1996.

[12] A. Satoh and K. Sugimoto, "An 1mi approach to gain parameter design for regional eigenvalue/eigenstructure assignment," in IEEE Conference on Decision and Control. IEEE, 2006, pp. 5778-5783.

[13] A. Caldas, A. Micaelli, M. Grossard, M. Makarov, P. RodriguezAyerbe, and D. Dumur, "Object-level impedance control for dexterous manipulation with contact uncertainties using an LMI-based approach," in IEEE International Conference on Robotics and Automation, 2015. (accepted).

[14] R. M. Murray and S. S. Sastry, A Mathematical Introduction to Robotic Manipulation. CRC press, 1994.

[15] J. Kerr and B. Roth, "Analysis of multifingered hands," The International Journal of Robotics Research, vol. 4, no. 4, pp. 3-17, 1986.

[16] A. Caldas, A. Micaelli, M. Grossard, M. Makarov, P. RodriguezAyerbe, and D. Dumur, "New metric for wrench space reachability of multifingered hand with contact uncertainties," in IEEE/ASME International Conference on Advanced Intelligent Mechatronics (AIM), July 2014, pp. 1236-1242.

[17] J. Lofberg, "Yalmip: A toolbox for modeling and optimization in matlab," in IEEE International Symposium on Computer Aided Control Systems Design, 2004, pp. 284-289.

[18] J. R. Flanagan and A. K. Rao, "Trajectory adaptation to a nonlinear visuomotor transformation: evidence of motion planning in visually perceived space," Journal of neurophysiology, vol. 74, no. 5, pp. 2174 2178,1995 